\title{
Experiencia con videocápsula endoscópica en el estudio y diagnóstico de enfermedades del intestino delgado en un hospital de alta complejidad en Medellín, Colombia
}

\section{Experience with video capsule endoscopy (VCE) in the study and diagnosis of small bowel diseases in a tertiary hospital in Medellín, Colombia}

Gabriel Alonso Mosquera-Klinger, MD, ${ }^{1}$ Joselyn Camargo Trillos, MD, ${ }^{2}$ Luz Adriana Gómez Ramírez, MD, ${ }^{3}$ Melissa Bustamante Duque, MD, ${ }^{3}$ Jhon Jaime Carvajal Gutiérrez, MD. ${ }^{1}$

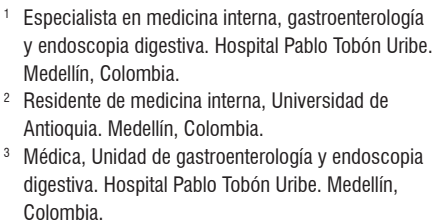
y endoscopia digestiva. Hospital Pablo Tobón Uribe. Medellín, Colombia.

2 Residente de medicina interna, Universidad de Antioquia. Medellín, Colombia.

3 Médica, Unidad de gastroenterología y endoscopia digestiva. Hospital Pablo Tobón Uribe. Medellín, Colombia.

\begin{abstract}
Resumen
Introducción: el intestino delgado (ID) es un órgano difícil de estudiar debido a su gran tamaño y ubicación anatómica; los estudios endoscópicos convencionales (esofagogastroduodenoscopia y colonoscopia) no logran evaluarlo en su totalidad y la enteroscopia con balón es más invasiva, con mayores efectos adversos y tasas de complicaciones. La videocápsula endoscópica (VCE) es una tecnología mínimamente invasiva que permite visualizar de forma dinámica y completa la mucosa del ID; es el método directo más seguro y con menos tasas de complicaciones. Metodología: se realizó un estudio descriptivo de corte transversal, para describir la experiencia clínica en la utilización de la VCE en un hospital de alta complejidad en Medellín, Colombia; asimismo, determinar las indicaciones, síntomas más comunes y hallazgos anormales. Resultados: se realizaron 374 VCE en el período de estudio: 282 ambulatorias $(75,4 \%)$ y 92 hospitalizados $(24,6 \%)$. En ambos grupos la indicación más frecuente fue sangrado digestivo oscuro $(38,65 \%$ y $53,26 \%$, respectivamente), seguidos por anemia crónica en el $27,65 \%$ de ambulatorios y por enfermedad inflamatoria intestinal (EII) en $21,74 \%$ de los hospitalizados. Los hallazgos anormales fueron más frecuentes en el yeyuno y las lesiones más comunes fueron angiodisplasias y lesiones inflamatorias. Conclusiones: en las indicaciones más frecuentes (sangrado digestivo oscuro y anemia) se documentaron potenciales fuentes de sangrado en más del $70 \%$ de los casos; siendo las lesiones vasculares e inflamatorias los hallazgos más comunes. En seguimiento o evaluación de Ell se documentaron en más del 50 \% lesiones inflamatorias. La VCE es muy segura, no se presentó ninguna complicación relacionada con la VCE.
\end{abstract}

Palabras clave

Cápsula endoscópica, enteroscopia, intestino delgado, sangrado digestivo oscuro.

\section{Abstract}

Introduction: The small intestine is a difficult organ to study due to its large size and anatomical location. Conventional esophagogastroduodenoscopy and colonoscopy cannot evaluate the entirety of the small intestine and balloon enteroscopy is more invasive, has more adverse effects, and has higher complication rates. Video capsule endoscopy (VCE) is a minimally invasive technology that allows a dynamic and complete view of the mucosa of the small intestine. It is the safest direct method which has the lowest complication rates. Methodology: This is a cross-sectional descriptive observational study that describes clinical experience in the use of VCE at a highly complex hospital in Medellin, Colombia. It also describes the indications for VCE, and the most common symptoms and abnormal findings. Results: VCE was used to study 282 outpatients (75.4\%) and 92 hospitalized patients $(24.6 \%)$ during the study period. In both groups, the most frequent indication was obscure digestive bleeding (38.65\% and $53.26 \%$ respectively), followed by chronic anemia in $27.65 \%$ of outpatients, and inflammatory bowel disease (IBD) in $21.74 \%$ of hospitalized patients. Abnormal findings were more frequent in the jejunum, and the most common lesions were angiodysplasias and inflammatory lesions. Conclusions: Potential sources of bleeding were documented in more than $70 \%$ of the cases of the most frequent indications which were obscure digestive bleeding and anemia. Vascular and inflammatory lesions were the most common findings. In follow-up evaluation of IBD, inflammatory lesions were documented in more than $50 \%$ of the cases. VCE is very safe, and there were no complications related to the VCE.

Keywords

Endoscopic capsule, enteroscopy, small intestine, dark digestive bleeding. 


\section{INTRODUCCIÓN}

\section{Planteamiento del problema y justificación}

El intestino delgado (ID) es un órgano ubicado en la parte media del tracto digestivo; debido a su tamaño (en promedio 6 metros), ubicación anatómica y características estructurales es difícil de inspeccionar a través de los estudios endoscópicos convencionales (esofagogastroduodenoscopia y colonoscopia). La exploración del ID mediante enteroscopia simple o de doble balón es más invasiva, genera más efectos adversos, tiene mayores tasas de complicaciones y es limitada en la evaluación inicial ya que se debe escoger muy bien la vía de ingreso (si es anterógrada o retrógrada) y la enteroscopia intraoperatoria requiere la realización de una laparoscopia o laparotomía abierta para el paso asistido quirúrgicamente del endoscopio a través del intestino, por lo cual resulta demasiado invasiva y aumenta la morbimortalidad.

La videocápsula endoscópica (VCE) es una tecnología mínimamente invasiva que permite visualizar de forma dinámica y completa la mucosa del ID; es fácil de utilizar, cómoda para el paciente y no requiere sedación en la mayoría de los casos. Desde su aprobación en el uso en humanos, las indicaciones de este estudio se han ido extendiendo y en la actualidad se reconocen múltiples indicaciones: sangrado digestivo de origen oscuro (oscuro manifiesto, oscuro oculto), anemia crónica, enfermedad de Crohn, tumores del ID, síndromes polipósicos hereditarios, enfermedad celíaca, diarrea crónica y dolor abdominal $(1,2)$. Adicionalmente, permite una adecuada ubicación de lesiones para determinar por cuál vía invasiva se realiza el diagnóstico histológico (enteroscopia anterógrada frente a enteroscopia retrógrada) $(2,3)$.

En agosto de 2001 la Food and Drug administration (FDA) aprobó la utilización de la VCE como método diagnóstico en enfermedades del ID en humanos. En Colombia el primer centro en adquirir esta tecnología fue la clínica Marly de Bogotá en el año 2003, y en el año 2009 se publicó su experiencia con 100 pacientes (4). La unidad de gastroenterología y endoscopia digestiva de nuestro centro está realizando VCE desde 2009. Inicialmente se usó la VCE de Olympus; en 2011 se publicó la experiencia de VCE en pacientes con sangrado digestivo oscuro (5). Desde el 2014 se viene usando la VCE PillCam SB 3 para la evaluación de patologías de ID tanto en el ámbito ambulatorio como en el hospitalizado.

Teniendo en cuenta que el Hospital Pablo Tobón Uribe (HPTU) es un centro de referencia de enfermedades digestivas de la región, se decidió presentar la experiencia en nuestra población atendida para determinar las indicaciones, factores de riesgo de la población estudiada y ubicación de lesiones documentadas en la cápsula; describir el tipo de lesiones y, de igual forma, evaluar si el resultado de la cápsula modificó de forma importante la conducta en quienes se encontraron hallazgos anormales.

\section{MATERIALES Y MÉTODOS}

Estudio descriptivo de corte transversal en el que se realizó la revisión de los registros de pacientes que fueron estudiados con VCE en el período comprendido entre enero de 2014 y diciembre de 2017. Posteriormente, se hizo la revisión detallada de las diferentes variables a medir a través de los datos obtenidos en el procedimiento y en la historia clínica del hospital o la historia traída por el paciente (en los casos ambulatorios que no se atendieron en nuestro centro). Los datos se registraron en una tabla de recolección en Excel para su posterior análisis estadístico.

En la Tabla 1 se pueden encontrar los datos demográficos; procedencia del estudio (ambulatorio u hospitalizado); las indicaciones y el síntoma principal de referencia del paciente. Los factores de riesgo tales como consumo de cigarrillo, alcohol, antiinflamatorios no esteroideos (AINE), glucosamina, medicamentos homeopáticos, anticoagulantes o antiagregantes, hepatopatía e historia de enfermedad renal crónica (ERC); la preparación y los hallazgos evidenciados en la cápsula se describen en el texto de los resultados.

Los criterios de inclusión fueron la edad mayor de 15 años y la firma del consentimiento informado institucional.

\section{Descripción del procedimiento}

Se utilizó videocápsula PillCam SB 3 de dimensiones 26,2 $\mathrm{mm} \times 11,4 \mathrm{~mm}$; peso: $3 \mathrm{~g}$, campo de visión 156 grados, tiempo estimado de capturas mayor de 8 horas. Después de la activación, la cápsula toma 2-6 fotos por segundo.

La preparación estándar para pacientes tanto ambulatorios como hospitalizados es con 2 sobres de polietilenglicol 3350 y 1 tableta masticable de simeticona que se inician desde el día previo; además, se exigió un período de ayuno de al menos 8 horas. Se colocaron 8 sensores en la pared abdominal conectados a la grabadora de datos que se encuentra ubicada en el cinturón que el paciente llevaba durante el tiempo del examen. La tecnología empleada fue given imagine.

Luego de sincronizar la cápsula con la grabadora de datos, se le dio a tomar la cápsula con 10-20 mL de agua, luego se mantenían en sala de fisiología digestiva hasta observar que la cápsula avanzara hasta el duodeno; en casos que después de 1 hora la cápsula no avanzara por sí misma, se procedió a avanzarla endoscópicamente. En casos de pacientes con trastorno deglutorio se pasó la cápsula mediante el dispositivo de avance. Luego se les dio instrucciones para tomar líquidos 1 hora después de la verificación del paso de la cápsula al ID, 4 horas después podían hacer ingesta de comida 
Tabla 1. Datos demográficos, clínicos e indicaciones de pacientes sometidos a VCE en el HPTU

\begin{tabular}{|c|c|c|c|}
\hline Variable & $\begin{array}{c}\text { Total } \\
\mathrm{n}: 374(100 \%)\end{array}$ & $\begin{array}{l}\text { Ambulatorios: } \\
282(100 \%)\end{array}$ & $\begin{array}{l}\text { Hospitalarios: } \\
92(100 \%)\end{array}$ \\
\hline \multicolumn{4}{|l|}{ Sexo } \\
\hline Femenino & $218(58,2 \%)$ & $169(59,9 \%)$ & $49(53,2 \%)$ \\
\hline Masculino & $155(41,8 \%)$ & $113(40,1 \%)$ & $43(46,8 \%)$ \\
\hline \multicolumn{4}{|l|}{ Indicación de la VCE } \\
\hline SDO & $159(42,4 \%)$ & $109(38,65 \%)$ & $50(54,3 \%)$ \\
\hline Anemia crónica & $92(24,5 \%)$ & $78(27,65 \%)$ & $14(15,2 \%)$ \\
\hline Diarrea crónica & $36(9,6 \%)$ & $32(11,3 \%)$ & $4(4,3 \%)$ \\
\hline Sospecha de Ell & $62(23,1 \%)$ & $42(14,8 \%)$ & $20(21,7 \%)$ \\
\hline Poliposis & $5(1,3 \%)$ & $5(1,7 \%)$ & $0(0 \%)$ \\
\hline Sospecha de neoplasia & $10(2,6 \%)$ & $9(3,1 \%)$ & $1(1 \%)$ \\
\hline Dolor abdominal & $9(2,4 \%)$ & $7(2,4 \%)$ & $2(2,1 \%)$ \\
\hline \multicolumn{4}{|l|}{ Síntomas } \\
\hline Ningún síntoma & $5(1 \%)$ & $5(1,77 \%)$ & $0(0 \%)$ \\
\hline Sangrado digestivo oscuro & $85(22 \%)$ & $45(15,95 \%)$ & $40(43,4 \%)$ \\
\hline Anemia crónica & $156(41 \%)$ & $134(47,52 \%)$ & $22(23,9 \%)$ \\
\hline Diarrea crónica & $69(18 \%)$ & $54(19,15 \%)$ & $15(16,4 \%)$ \\
\hline Perdida inexplicada de peso & $23(6,1 \%)$ & $23(8,15 \%)$ & $0(0 \%)$ \\
\hline Dolor abdominal & $20(5,3 \%)$ & $16(5,67 \%)$ & $4(4,3 \%)$ \\
\hline Aftas orales recurrentes & $3(0,8 \%)$ & $2(0,7 \%)$ & $1(0,1 \%)$ \\
\hline No hay datos & $3(0,8 \%)$ & $3(0,1 \%)$ & $0(0 \%)$ \\
\hline
\end{tabular}

EII: enfermedad inflamatoria intestinal; SDO: sangrado digestivo oscuro; VCE: videocápsula endoscópica.

normal y realizar actividades cotidianas, $\mathrm{y}$ en un promedio de 8 a 10 horas se retiró la grabadora.

En todos los casos, el video se evaluó exhaustivamente por el especialista encargado a una velocidad de 5 fotos/ segundo, y en caso de dudas en los hallazgos, se revisaban y discutían con un segundo operador.

\section{Forma de recolección y análisis de datos}

Los investigadores realizaron la recolección de las variables en una base de datos en Excel. Se utilizó estadística descriptiva para las variables categóricas (nominales u ordinales) mediante distribuciones de frecuencias y porcentuales, y para las variables numéricas mediante medidas de tendencia central como el promedio y la mediana.

\section{Población y muestra}

Pacientes mayores de 15 años, a quienes se les realizó VCE en el HPTU de la ciudad de Medellín, Colombia.

\section{Consideraciones éticas}

El protocolo se presentó al comité de ética del HPTU, donde se evaluó y aprobó. Se siguieron las recomendacio- nes éticas internacionales y nacionales, como la declaración de Helsinki y la resolución 8430 de 1993 del Ministerio de Salud de Colombia, que clasifica al estudio como de riesgo mínimo, ya que no pone en riesgo la integridad del paciente.

\section{RESULTADOS}

Se realizó un total de 374 estudios de VCE en el período analizado: 282 fueron ambulatorios (169 mujeres, 113 hombres) y 92 pacientes (49 mujeres, 43 hombres) fueron en el contexto de hospitalización. El promedio de edad fue de 56,5 con un rango entre 15-92 años.

La preparación del ID se consideró adecuada en 79/92 $(85,87 \%)$ de los hospitalizados y en $258 / 282$ (91,49\%) de los ambulatorios. Tanto en pacientes ambulatorios como hospitalizados, la indicación más frecuente fue sangrado digestivo oscuro, que se observó en 109/282 (38,65\%) de los ambulatorios y en 50/92 (53.26\%) de los hospitalizados, seguidos por anemia crónica en 78/282 (27,65 \%) de los ambulatorios y el seguimiento o diagnósticos de pacientes con enfermedad de Crohn en 20/92 (21,74\%) de los hospitalizados.

En cuanto a los factores de riesgo, en pacientes hospitalizados se identificó que de los relacionados con sangrado digestivo se presentaron: hábito de fumar 17/92 (18,48 
$\%)$, antiagregantes/antiplaquetarios en $15 / 92(16,30 \%)$, AINE en 7/92 (7,60 \%) y alcohol en 3/92 (3,26\%); en 1 paciente con glucosamina se identificó una úlcera con estenosis inflamatoria severa (Figura 1A). En los pacientes ambulatorios se presentó: hábito de fumar en 40/282 (14 $\%$ ), alcohol en $26 / 282$ (9\%), antiagregante/anticoagulante en $28 / 282(10 \%)$, AINE en $25 / 282(8,8 \%)$, glucosamina en $4 / 282(1,4 \%)$, homeopáticos en $1 / 282$, hepatopatía crónica en 9/282 (3.1\%) y ERC en 13/282 (4,6\%).

Los síntomas referidos por los pacientes ambulatorios fueron, en orden de frecuencia, los siguientes: anemia en 134 (47,52 \%), diarrea crónica en 54 (19,15\%), sangrado digestivo manifiesto en 45 (15,95\%), pérdida inexplicable de peso en $23(8,15 \%)$ y dolor abdominal en $16(5,67 \%)$. En pacientes hospitalizados, los síntomas más frecuentes fueron sangrado en $49(53,26 \%)$, anemia en $22(23,91 \%)$, diarrea en $15(16,30 \%)$, dolor abdominal en $4(4,35 \%)$ y aftas orales recurrentes en $1(1,08 \%)$.

Sobre estudios endoscópicos previos, a 86/92 pacientes hospitalizados (el 93,5\%) se les había realizado endoscopia previamente y $81 / 92$ ( $88 \%$ ) tenían colonoscopia.

De los pacientes hospitalizados, a $18(19,56 \%)$ se les realizó enteroscopia en relación con el hallazgo anormal documentado en VCE; de estos, 10 tenían lesiones vascula-

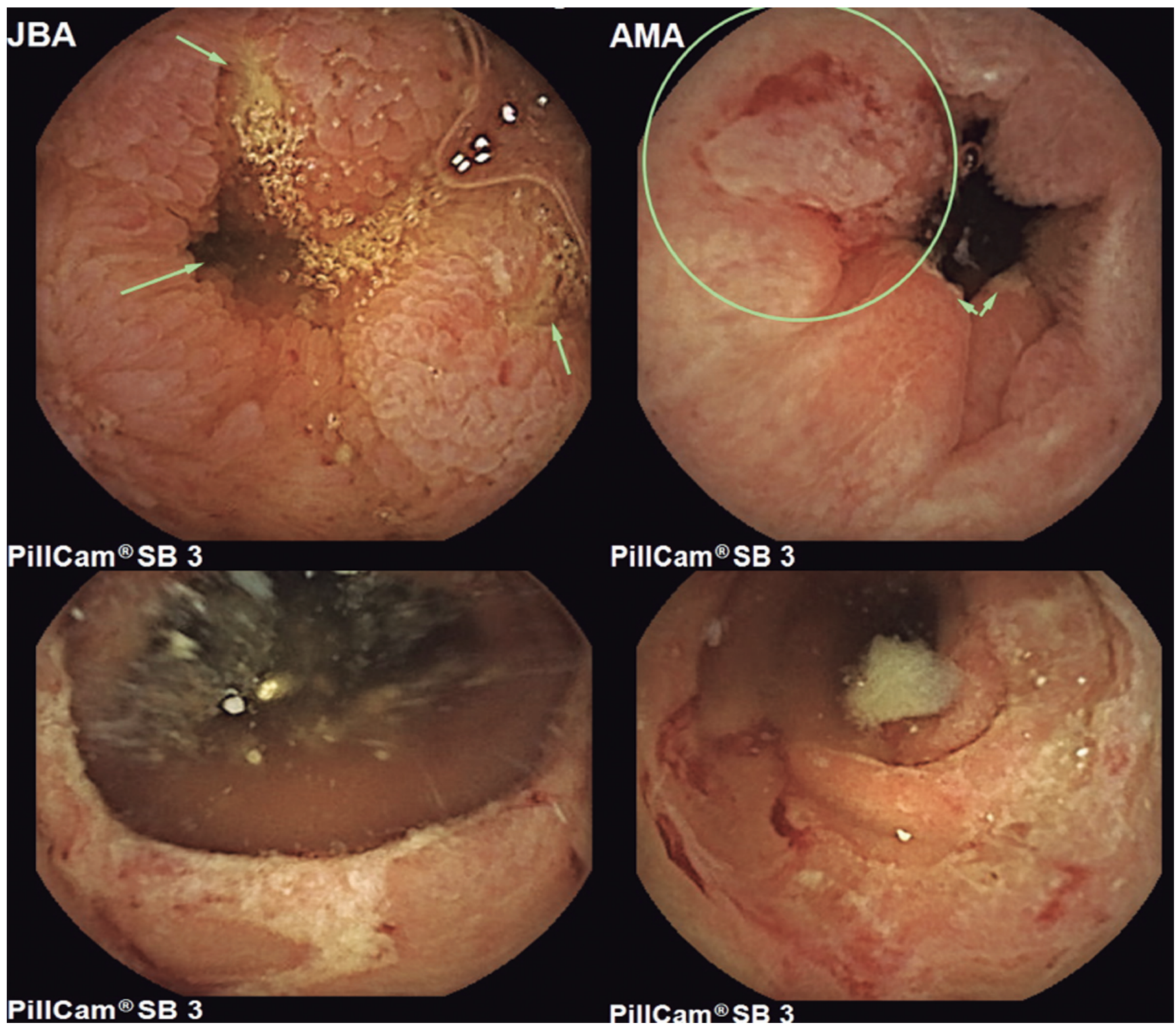

Figura 1. A. Enteritis ulcerada con estenosis por consumo de glucosamina. B. Ileítis ulcerada en paciente con enfermedad de Crohn. C. Úlcera inflamatoria hemicircunferencial en íleon en paciente con enfermedad de Crohn. D. Úlceras inflamatorias en yeyuno con sangrado activo. 
res dadas por angiodisplasias ( 5 de ellos con diagnóstico de Osler Weber Rendú) a los cuales se les realizó la enteroscopia con fines terapéuticos, a todos se les realizó terapia con argón plasma; a los otros 8 pacientes se les encontró lesiones inflamatorias y/o tumorales, por lo cual se les realizó enteroscopia para la toma de biopsias y marcación de la lesión.

En ambos grupos, cuando la indicación del procedimiento fue sangrado digestivo oscuro, de los 159 casos, en $73(45,91 \%)$ fue por causas vasculares y $53(33,33 \%)$ por lesiones inflamatorias. Cuando la indicación fue anemia, de los 93 casos, las fuentes potenciales de sangrado se identificaron en más del $70 \%$ de los casos, lesiones vasculares en $36(38,71 \%)$, seguidas por inflamatorias $26(27,95 \%)$ y tumorales $6(6,45 \%)$. Cuando la indicación estuvo relacionada con el diagnóstico y seguimiento de enfermedad inflamatoria intestinal, de los 62 casos, los hallazgos encontrados fueron $34(54,83 \%)$ inflamatorios (Figuras 1B-1D), seguidos por vasculares en $7(11,29 \%)$ de casos.

En pacientes hospitalizados, la localización más frecuente de los hallazgos anormales fue en yeyuno 43/92 $(46,7 \%)$; de estos pacientes, las lesiones más frecuentes fueron angiodisplasias en 32/43 (74,4\%) e inflamatorias en 18/43 (41,8\%). El duodeno fue la segunda localización con más hallazgos anormales: 39/92 (42,3\%), en los que predominaron las angiodisplasias en $27 / 39(69,2 \%)$ y luego las inflamatorias 17/39 (43,5\%) (Tabla 2).

En cuanto a la evidencia de lesiones tumorales, se documentaron 12 lesiones, 6 de estas fueron pólipos de aspecto benigno en estómago y duodeno; un paciente tuvo una lesión gástrica correspondiente a neoplasia gástrica y el resto tuvo lesiones subepiteliales ulceradas que fueron en relación con tumores neuroendocrinos en yeyuno e íleon.

Tabla 2. Ubicación y tipo de lesiones en VCE realizadas en el HPTU

\begin{tabular}{lccc}
\hline $\begin{array}{l}\text { Ubicación de lesiones/tipo de } \\
\text { lesiones }\end{array}$ & Total & Ambulatorios: & Hospitalarios: \\
$\mathbf{n}: \mathbf{3 7 4}(\mathbf{1 0 0} \%)$ & $\mathbf{2 8 2 ( 1 0 0 \% )}$ \\
\hline Esófago & $3(0,80 \%)$ & $3(1,06 \%)$ & $0(0 \%)$ \\
Angiodisplasias & $2(0,53 \%)$ & $2(0,70 \%)$ & $0(0 \%)$ \\
Erosión & $1(0,26 \%)$ & $1(0,35 \%)$ & $0(0 \%)$ \\
Estómago & $64(17.11 \%)$ & $46(16.31 \%)$ & $18(19.56 \%)$ \\
Lesiones vasculares & $26(6,95 \%)$ & $21(7,44 \%)$ & $5(5,43 \%)$ \\
Lesiones inflamatorias & $45(12,03 \%)$ & $34(12,05 \%)$ & $11(11,95 \%)$ \\
Neoplasia & $8(2,13 \%)$ & $5(1,77 \%)$ & $3(3,26 \%)$ \\
Linfangiectasia & $10(2,67 \%)$ & $10(3,54 \%)$ & $0(0 \%)$ \\
Atrofia & $3(0,8 \%)$ & $3(1 \%)$ & $0(0 \%)$ \\
Duodeno & $111(29,67 \%)$ & $72(25,53 \%)$ & $39(42,39 \%)$ \\
Hemolinfangiectasia & $19(5,08 \%)$ & $18(6,38 \%)$ & $1(0,1 \%)$ \\
Angiodisplasia & $68(18,18 \%)$ & $41(14,53 \%)$ & $27(29,34 \%)$ \\
Lesiones inflamatorias & $51(13,63 \%)$ & $34(12,05 \%)$ & $17(18,47 \%)$ \\
Lesiones tumorales & $10(2,67 \%)$ & $8(2,83 \%)$ & $2(2,17 \%)$ \\
Atrofia de mucosas & $5(1,33 \%)$ & $3(1,06 \%)$ & $2(2,17 \%)$ \\
Diverticulosis & $3(0,8 \%)$ & $1(0,35 \%)$ & $2(2,17 \%)$ \\
Yeyuno & $159(42,51 \%)$ & $116(41,13 \%)$ & $43(46,74 \%)$ \\
Linfangiectasia & $18(4,81 \%)$ & $16(5,67 \%)$ & $2(2,17 \%)$ \\
Angiodisplasia & $99(37,02 \%)$ & $67(23,75 \%)$ & $32(34,78 \%)$ \\
Lesión inflamatoria & $75(20,05 \%)$ & $57(20,21 \%)$ & $18(19,56 \%)$ \\
Lesiones tumorales & $5(2,13 \%)$ & $3(1,06 \%)$ & $5(5,43 \%)$ \\
Cambios de atrofia & $3(0,80 \%)$ & $2(0,70 \%)$ & $1(1,08 \%)$ \\
Divertículo & $1(0,26 \%)$ & $1(0,35 \%)$ & $0(0 \%)$ \\
Íleon & $111(29,67 \%)$ & $79(28,01 \%)$ & $32(34,78 \%)$ \\
Linfangiectasia & $7(1,87 \%)$ & $6(2,12 \%)$ & $1(1,08 \%)$ \\
Lesiones vasculares & $51(13,63 \%)$ & $32(11,34 \%)$ & $19(20,65 \%)$ \\
Lesiones inflamatorias & $70(18,71 \%)$ & $50(17,73 \%)$ & $20(21,73 \%)$ \\
Lesiones tumorales & $6(1,60 \%)$ & $3(1,06 \%)$ & $1(3,26 \%)$ \\
Atrofia & $3(1,80 \%)$ & $2(0,70 \%)$ & $08 \%)$ \\
Divertículo & $1(0,26 \%)$ & $1(0,35 \%)$ & $0(0 \%)$ \\
\hline
\end{tabular}




\section{DISCUSIÓN}

En la actualidad se dispone de múltiples técnicas para la evaluación del ID, estas incluyen VCE, enteroscopia con balón, enterografía por tomografía axial computarizada (TAC) multifásica (enteroTAC), enterografía por resonancia magnética (enteroRMN) y, en raras ocasiones, enteroscopia intraoperatoria. Estas modalidades pueden reconocer la mayoría de lesiones del ID y pueden impactar en las estrategias terapéuticas, a menudo evitando intervenciones quirúrgicas innecesarias o disminuyendo los tiempos quirúrgicos por una mejor localización de la lesión (1-3, 6). A pesar de estos avances en el estudio del ID, el enfoque más eficiente para el manejo de pacientes con sospecha de sangrado de ID no se ha determinado totalmente, pero la recomendación más fuerte en la actualidad es abordar mediante $\operatorname{VCE}(1,2,6)$. Debido a todo este crecimiento en métodos diagnósticos para explorar el ID, una guía clínica publicada recientemente recomienda el cambio del término sangrado digestivo oscuro a sangrado del ID, reservando el término sangrado digestivo oscuro al sangrado que no pueda identificarse después de finalizar toda la evaluación del tracto gastrointestinal (7).

En el trabajo de Maeda y colaboradores se demostró que la sensibilidad y el valor predictivo negativo del VCE fueron del $100 \%$, tanto por la presencia de lesiones en el ID como por el abordaje terapéutico en los casos con lesiones significativas. En dicho estudio, la VCE nunca pasó por alto los hallazgos relevantes en el ID, y un dato de gran interés es que los hallazgos negativos de una VCE casi siempre corresponden a una inadecuada indicación (3). En una revisión sistemática y metaanálisis reciente se demostró que la VCE negativa proporciona información sobre un bajo riesgo de resangrado a futuro. Por tanto, estos pacientes podrían ser dados de alta y tratados de forma segura solo con conducta expectante (8). Sin embargo, en una carta al editor sobre ese trabajo se hace mención de que los resultados negativos del examen nunca deben tranquilizar al médico tratante; por el contrario, se recomienda una monitorización estrecha y un umbral bajo para repetir la endoscopia con el fin de identificar posibles lesiones no relacionadas con el ID en pacientes con sangrado manifiesto (9). Los pacientes que vuelven a sangrar después de 2 años podrían necesitar estudios adicionales nuevamente (8).

En los datos obtenidos en nuestro trabajo el rendimiento diagnóstico fue bueno, ya que en las 2 indicaciones más frecuentes (sangrado digestivo y anemia) se documentaron causas potenciales en más del $70 \%$ de los estudios, Un metaanálisis reciente encontró que el rendimiento diagnóstico combinado de la VCE en pacientes con sangrado digestivo oscuro (manifiesto u oculto) fue del 61,7 \% (10); sin embargo, hay varios factores que influyen en la proba- bilidad de encontrar la lesión causante del sangrado, entre los que se encuentran la presencia de sangrado activo, caída en más de $3 \mathrm{~g}$ en la hemoglobina y el tiempo de realización del estudio con respecto al último día de sangrado. Estos factores hacen que el rendimiento del estudio pueda variar entre un $11 \%$ y $87 \%(1,6)$.

En los pacientes de nuestro estudio con sospecha o diagnóstico previo de enfermedad inflamatoria intestinal se identificaron lesiones inflamatorias en el $50 \%$ de los casos. Aunque hasta el $66 \%$ de los pacientes con enfermedad de Crohn tiene compromiso del ID al momento del diagnóstico y que la mayoría (hasta el $90 \%$ ) tiene compromiso en íleon distal (11), algunos estudios han mostrado que la evaluación del ID con VCE aporta entre un $22 \%-24 \%$ en el diagnóstico de la enfermedad de Crohn ya sea por lesiones no evidenciadas en la colonoscopia y estudios imagenológicos o por la imposibilidad de ingresar al íleon $(12,13)$, lo que convierte a la VCE en un método complementario ideal en pacientes que tienen síntomas compatibles con Enfermedad de Crohn, elevación de reactantes de fase aguda o elevación de calprotectina fecal en los que no se haya logrado realizar un diagnóstico certero de la enfermedad y/o de su extensión. Inclusive reclasificando pacientes que inicialmente tenían el diagnóstico de una colitis ulcerativa o una colitis indeterminada y que ante la evidencia de lesiones en el ID deban ser manejados como si tuvieran enfermedad de Crohn. Además, conocer el grado de compromiso del ID puede tener implicaciones en el tratamiento, seguimiento y pronóstico de la enfermedad. Finalmente, permite realizar el seguimiento de lesiones conocidas para evaluar la respuesta al tratamiento, definiendo en algunos la remisión profunda o necesidad de modificaciones en la terapia de mantenimiento (14).

La cápsula fue una herramienta fundamental para la toma de decisiones diagnósticas y terapéuticas en relación con los hallazgos anormales. En casi el $20 \%$ de pacientes hospitalizados por sangrado digestivo oscuro se realizó enteroscopia de doble balón (con fines diagnósticos o terapéuticos: aplicación de argón plasma en lesiones vasculares, toma de biopsias y marcación de tumores para resección). Se observó que en todos los casos de enteroscopia la cápsula fue la guía para definir la vía de ingreso del enteroscopio (anterógrada o retrograda) por la ubicación topográfica, como lo anotado en la literatura $(1-3,6,7)$.

En pacientes hospitalizados, a 86/92 (el 93,5\%) se les realizó endoscopia previamente y a 81/92 (88 \%) colonoscopia. En nuestro estudio no todos tuvieron endoscopia y/o colonoscopia antes de la cápsula, ya que algunos pacientes fueron remitidos de otros centros de la ciudad para realizarse la VCE; además, en nuestra serie la sospecha diagnóstica de EC con compromiso en ID fue la segunda causa de exámenes en hospitalizados. En estos pacientes, la 
realización de la cápsula es con fines de establecer la extensión de la enfermedad y el compromiso del ID (10). Por esta razón, estos datos difieren de lo reportado por la Dra. Galiano y colaboradores, en cuya cohorte el $100 \%$ de los pacientes tenía endoscopia digestiva superior y colonoscopia antes del estudio con VCE (4). Aunque sí estamos de acuerdo y nos adherimos a las recomendaciones de realizar estudios básicos iniciales en especial a pacientes con sangrado digestivo oscuro o anemia crónica con sospecha de pérdidas gastrointestinales.

Los riesgos inherentes al procedimiento son bajos; se ha descrito en especial la retención de la VCE, aunque esto ocurre en alrededor del $1 \%$ de los casos (4). En nuestra serie no se presentó ninguna complicación. Cuando hay síntomas obstructivos como vómito asociado con distensión y/o dolor abdominal, se recomienda la realización del estudio del ID con enteroTAC o enteroRMN según la experiencia o disponibilidad institucional, pues son estos pacientes los que tienen mayor riesgo de retención de la cápsula (6).

En conclusión, de las múltiples indicaciones de la VCE en nuestro centro, el sangrado digestivo oscuro y la anemia fueron las más frecuentes, con un rendimiento diagnóstico que superó el $70 \%$. En pacientes hospitalizados se utilizó la VCE para el estudio y la evaluación de los pacientes con sospecha o diagnóstico conocido de enfermedad de Crohn, esta última es una indicación que aporta gran información del diagnóstico, clasificación, pronóstico y seguimiento en este tipo de pacientes.

La VCE es segura, no se presentaron complicaciones relacionadas con el procedimiento y es una herramienta de gran utilidad para definir la vía de entrada y el abordaje inicial de un sangrado digestivo. Hasta ahora es la casuística más grande publicada en Colombia. Se recomienda hacer estudios multicéntricos en nuestro medio para conocer mejor nuestra prevalencia e incidencia de lesiones en ID y para conocer las características operativas de este método en nuestra población.

\section{REFERENCIAS}

1. Enns RA, Hookey L, Armstrong D, Bernstein CN, Heitman SJ, Teshima C, et al. Clinical Practice Guidelines for the Use of Video Capsule Endoscopy. Gastroenterology. 2017;152(3):497-514. doi: 10.1053/j.gastro.2016.12.032.

2. ASGE Standards of Practice Committee, Gurudu SR, Bruining DH, Acosta RD, Eloubeidi MA, Faulx AL, et al. The role of endoscopy in the management of suspected small-bowel bleeding. Gastrointest Endosc. 2017;85(1):2231. doi: 10.1016/j.gie.2016.06.013.

3. Maeda Y, Moribata K, Deguchi H, Inoue I, Maekita T, Iguchi $\mathrm{M}$, et al. Video capsule endoscopy as the initial examination for overt obscure gastrointestinal bleeding can efficiently identify patients who require double-balloon enteroscopy.
BMC Gastroenterol. 2015;15:132. doi: 10.1186/s12876015-0362-7.

4. Galiano MT, Sánchez F, Pineda LF. Experiencia clínica del uso de la videocápsula endoscópica en el diagnóstico de patología del intestino delgado. Rev Col Gastroenterol. 2009;24(1):17-25.

5. Juliao F, Muñoz E, Yepes C. Diagnostic performance of capsule endoscopy and findings from patients with obscure gastrointestinal bleeding at the Hospital Pablo Tobon Uribe in Medellin, Colombia. Rev Col Gastroenterol. 2013;28(2):109-17.

6. Pennazio M, Spada C, Eliakim R, Keuchel M, May A, Mulder CJ, et al. Small-bowel capsule endoscopy and device-assisted enteroscopy for diagnosis and treatment of small-bowel disorders: European Society of Gastrointestinal Endoscopy (ESGE) Clinical Guideline. Endoscopy. 2015;47(4):35276. doi: 10.1055/s-0034-1391855.

7. Gerson LB, Fidler JL, Cave DR, Leighton JA. ACG Clinical Guideline: Diagnosis and Management of Small Bowel Bleeding. Am J Gastroenterol. 2015;110(9):1265-87; quiz 1288. doi: 10.1038/ajg.2015.246.

8. Yung DE, Koulaouzidis A, Avni T, Kopylov U, Giannakou A, Rondonotti E, et al. Clinical outcomes of negative smallbowel capsule endoscopy for small-bowel bleeding: a systematic review and meta-analysis. Gastrointest Endosc. 2017;85(2):305-317.e2. doi: 10.1016/j.gie.2016.08.027.

9. Van de Bruaene C, Hindryckx P, De Looze D. Capsule endoscopy in obscure GI bleeding: better to be negative, but let us not be too positive. Gastrointest Endosc. 2017;86(3):5745. doi: 10.1016/j.gie.2017.04.012.

10. Teshima CW, Kuipers EJ, van Zanten SV, Mensink PB. Double balloon enteroscopy and capsule endoscopy for obscure gastrointestinal bleeding: an updated meta-analysis. J Gastroenterol Hepatol. 2011;26(5):796-801. doi: 10.1111/j.1440-1746.2010.06530.x.

11. Van Assche G, Dignass A, Panes J, Beaugerie L, Karagiannis $\mathrm{J}$, Allez M, et al. The second European evidence-based Consensus on the diagnosis and management of Crohn's disease: Definitions and diagnosis. J Crohns Colitis. 2010 Feb;4(1):7-27. doi: 10.1016/j.crohns.2009.12.003.

12. Dionisio PM, Gurudu SR, Leighton JA, Leontiadis GI, Fleischer DE, Hara AK, et al. Capsule endoscopy has a significantly higher diagnostic yield in patients with suspected and established small-bowel Crohn's disease: a meta-analysis. Am J Gastroenterol. 2010;105(6):1240-8; quiz 1249. doi: 10.1038/ajg.2009.713.

13. Adler SN, Yoav M, Eitan S, Yehuda C, Eliakim R. Does capsule endoscopy have an added value in patients with perianal disease and a negative work up for Crohn's disease? World J Gastrointest Endosc. 2012;4(5):185-8. doi: 10.4253/wjge. v4.i5.185.

14. Gomollón F, Dignass A, Annese V, Tilg H, Van Assche G, Lindsay JO, et al. 3rd European Evidence-based Consensus on the Diagnosis and Management of Crohn's Disease 2016: Part 1: Diagnosis and Medical Management. J Crohns Colitis. 2017;11(1):3-25. doi: 10.1093/ecco-jcc/jjw168. 